\title{
PATH PROBABILITY OF RANDOM FRACTIONAL SYSTEMS DEFINED BY WHITE NOISES IN COARSE-GRAINED TIME. APPLICATION OF FRACTIONAL ENTROPY
}

\begin{abstract}
GUY JUMARIE
Abstract. One considers a class of fractional random processes defined as non-random dynamics subject to Gaussian white noises in coarse-grained time, according to Maruyama's notation. After some prerequisites on modified Riemann-Liouville fractional derivative, fractional Taylor's series and integration with respect to $(d x)^{\alpha}$, one displays the main results which are as follows: firstly, a general scheme to obtain the path probability density (in Feynman's sense) of some fractional stochastic dynamics; secondly an approximation, via Itô's lemma, for their characteristic functions, therefore approximate expressions for their path probability density; and thirdly, an approach via the maximum entropy principle (MEP) which holds when the dynamical equations of the state moments are available. One first uses the MEP combined with Shannon entropy, and then one applies the MEP with a new concept of fractional entropy which takes account of defects in observation. As a last application, one uses an optimization of distributed entropy based on fractional Fokker-Planck equation. All the paper is based on the modified RiemannLiouville derivative and the generalization of the Maruyama notation for Brownian motion, and the mathematics so involved is customarily referred to as physical mathematics or engineering mathematics.
\end{abstract}

Mathematics subject classification (2010): 60G22 60J65, 28A80, 26A33, 53-XX.

Keywords and phrases: Modified Riemann-Liouville derivative, fractional Taylor's series, fractional stochastic processes, path probability density, maximum entropy principle, distributed optimization, entropy of fractional order..

\section{REFERENCES}

[1] M. AL-AKaidi, Fractal Speech Processing, Cambridge University press, 2004.

[2] V.V. ANH AND N. N. LEONENKo, Spectral theory of renormalized fractional random fields, Teor. Imovirnost. ta Matem. Statyst, 66 (2002), 3-14.

[3] L.M.C. CAMPOS, On a concept of derivative of complex order with applications to special functions, IMA J. Appl. Math., 33 (1984), 109-133.

[4] L.M.C. CAmpos, Fractional calculus of analytic and branched functions, in R.N. Kalia (Ed.), Recent Advances in Fractional Calculus, Global Publishing Company, 1993.

[5] M. CAPUTO, Linear model of dissipation whose $Q$ is almost frequency dependent II, Geophys. J. R. Ast. Soc., 13 (1967), 529-539.

[6] R. CARPinteri And P. Mainardi (eds.), Fractals and Fractional Calculus in Continuum, Mechanics, CISM Lecture Notes, Vol. 378, 1997.

[7] M.M. DJRbAShiAn AND A.B. Nersesian, Fractional derivative and the Cauchy problem for differential equations of fractional order (in Russian), Izv. Acad. Nauk Armjanskoi SSR, 3, 1 (1968), 3-29.

[8] K. FALCONER, Techniques in Fractal Geometry, Wiley, New York, 1997.

[9] R. Hilfer, Fractional time evolution, Applications of Fractional Calculus in Physics (R. Hilfer, ed.), World Scientific, Singapore, 2000, 87-130.

[10] F. HuAng AND F. LiU, The space-time fractional diffusion equation with Caputo derivatives, J. Applied Math. and Computing, 19, 1-2 (2005), 179-190. 
[11] E.T. JAYNES, Information theory and statistical mechanics, Phys. Rev., 106 (1957), 620-630; 108 (1957), 171-190.

[12] G. JUMARIE, Stochastic differential equations with fractional Brownian motion input, Int. J. Syst. Sc., 24, 6 (1993), 1113-1132.

[13] G. Jumarie, Maximum Entropy, Information Without Probability and Complex Fractals, , Fundamental Theories of Physics Series, Kluwer Academic Publishers, Dordrecht, Boston, London, 270 pp., 2000.

[14] G. Jumarie, Further results on Fokker-Planck equation of fractional order, Chaos, Solitons and Fractals, 12 (2001), 1873-1886.

[15] G. JumARIE, On the representation of fractional Brownian motion as an integral with respect to $(d t)^{\alpha}$, Applied Mathematics Letters, 18 (2005), 739-748.

[16] G. JUMARIE, On the solution of the stochastic differential equation of exponential growth driven by fractional Brownian motion, Applied Mathematics Letters, 18 (2005), 817-826.

[17] G. JUmARIE, Modified Riemann-Liouville derivative and fractional Taylor series of non-differentiable functions Further results, Computers and Mathematics with Applications, 51 (2006), 1367-1376.

[18] G. JUMARIE, New stochastic fractional models for Malthusian growth, the Poissonian birth prodess and optimal management of populations, Mathematical and Computer Modelling, 44 (2006), 231-254.

[19] G. JUMARIE, Probability calculus of fractional order and fractional Taylor's series. Application to Fokker-Planck equation and information of non-random functions, Chaos, Solitons and Fractals, 40, 3 (2009), 1428-1448.

[20] G. JUMARIE, Lagrangian mechanics of fractional order, Hamilton-Jacobi fractional PDE and Taylor's series of nondifferentiable functions, Chaos, Solitons and Fractals, 32, 3 (2007), 969-987.

[21] G. JUMARIE, Modeling fractional stochastic systems as non-random fractional dynamics driven by Brownian motion, Applied Mathematical Modelling, 32 (2008), 836-859.

[22] G. JumARIE, On some similarities and differences between fractional probability density, signed measure of probability and quantum probability, Modern Physics Letters, 23, 6 (2009), 1-15.

[23] H. Kober, On fractional integrals and derivatives, Quart. J. Math. Oxford, 11 (1940), 193-215.

[24] K.M. Kolwankar, A.D. Gangal, Holder exponents of irregular signals and local fractional derivatives, Pramana J. Phys., 48 (1997), 49-68.

[25] K.M. Kolwankar, A.D. Gangal, Local fractional Fokker-Planck equation, Phys. Rev. Lett., 80 (1998), 214-217.

[26] A.V. Letnivov, Theory of differentiation of fractional order, Math. Sb., 3 (1868), 1-7.

[27] S.A. Levin (ED.), Frontiers in Mathematical Biology, Springer-Verlag, New York, 1994.

[28] J. Liouville, Sur le calcul des differentielles à indices quelconques (in french), J. Ecole Polytechnique, 13 (1832), 71.

[29] B.B. Mandelbrot AND J.W. VAn Ness, Fractional Brownian motions, fractional noises and applications, SIAM Rev., 10 (1968), 422-437.

[30] B.B. MANDELbRot, The Fractal Geometry of Nature, W.H.Freeman, New York, 1982.

[31] B.B. Mandelbrot, Fractals and Scaling in Finance: Discontinuity, Concentration, Risk, Springer Verlag, New York, 1997.

[32] G. Maruyama, Continuous Markov processes and stochastic equations, Rend. Cir. Mat. Palermo, 2, 4 (1955), 48-90.

[33] K.S. Miller AND B. Ross, An Introduction to the Fractional Calculus and Fractional, Differential Equations, Wiley, New York, 1973.

[34] Y. MishurA, Stochastic calculus for fractional Brownian motion and related process, Springer Berlin, 2008.

[35] K. Nishimoto, Fractional Calculus, Descartes Press Co., Koroyama, 1989.

[36] K.B. Oldham And J. Spanier, The Fractional Calculus. Theory and Application Differentiation and Integration to Arbitrary Order, Academic Press, New York, 1974.

[37] T.J. OSLER, Taylor's series generalized for fractional derivatives and applications, SIAM. J. Mathematical Analysis, 2, 1 (1971), 37-47.

[38] I. Podlubny, Fractional Differential Equations, Academic Press, San Diego, 1999.

[39] A. Renyi, On measures of information and entropy, Proceedings of the $4^{\text {th }}$ Berkeley symposium on Mathematics, Statistics and Probability, 1 (1960), 547-561, University of California at Berkeley.

[40] B. Ross, Fractional Calculus and its Applications, Lecture Notes in Mathematics, Vol. 457, Springer, Berlin, 1974. 
[41] S.G. Samko, A.A. Kilbas, And O.I. Marichev, Fractional Integrals and derivatives. Theory and Applications, Gordon and Breach Science Publishers, London, 1987.

[42] M. SHAHER AND Z.M. OdiBAT, Fractional Green function for linear time-fractional inhomogeneous partial differential equations in fluid mechanics, J. Applied Math. and Computing, 24, 1-2 (2007), 167-178.

[43] C.E. Shannon, A mathematical theory of communication, Bell System Technical J., 27 (1948), 379423, 623-656.

[44] C. Ts allis, Possible generalization of Boltzmann-Gibbs statistics, J. Statist. Phys., 52*, 1-2 (1988), 479-487. 